\title{
Combined endoscopic third ventriculostomy and tumor biopsy in the management of pineal region tumors, safety considerations
}

\author{
Mohamed Amer Ibrahim Amer ${ }^{*}$ (i) and Hytham Ibrahim Shokry Elatrozy
}

\begin{abstract}
Background: Pineal region tumor is considered an ideal indication for endoscopic third ventriculostomy (ETV), with success rate up to $92 \%$. The ultimate advantage is the ability to obtain tumor biopsy for histopathological diagnosis and for further treatment strategy plan design.

Objectives: To investigate the safety considerations to minimize tumor biopsy-related bleeding, increase diagnostic accuracy, and establish a successful ETV procedure.

Methods: Retrospective study including 25 patients of pineal region tumor with concomitant triventricular hydrocephalus. Preoperative planning to perform ETV and obtain tumor biopsy was achieved either through single more anteriorly located precoronal burr-hole (17 patients) or two separate burr-holes (eight patients). The patients' age ranged from 9 months to 65 years with a mean age of 26.5 years. Preoperative MRI brain with coronal T2- and sagittal T1-weighted images was used to design trajectory.
\end{abstract}

Results: No significant intraventricular bleeding reported. A diagnostic tumor biopsy yield was successful in 22 patients. Ventriculoperitoneal shunt insertion was required in 1 patient failed to improve.

Conclusion: Endoscopic tumor biopsy is relatively safe with high diagnostic yield that helps in management protocol plus shunt independent control of associated hydrocephalus.

Keywords: Pineal tumor, Hydrocephalus, Endoscopic third ventriculostomy, Biopsy

\section{Introduction}

Pineal body tumors are histopathologically heterogenic group, including germ cell tumors, pineal tumors, astrocytic tumors, and cystic lesions. The clinical findings are quite consistent where approximately $90 \%$ of patients present with obstructive hydrocephalus and manifestation of raised intracranial pressure [1].

Initial step in the management should achieve control of hydrocephalus and pathological verification. Further treatment of the tumor can be planned afterwards, chemotherapy, radiotherapy, or surgical excision [2].

\footnotetext{
* Correspondence: m_amer_2000@yahoo.com

Faculty of medicine, Neurosurgical Department, Tanta University Hospitals, Tanta, Egypt
}

\section{Springer Open}

(c) The Author(s). 2018 Open Access This article is distributed under the terms of the Creative Commons Attribution 4.0 International License (http://creativecommons.org/licenses/by/4.0/), which permits unrestricted use, distribution, and reproduction in any medium, provided you give appropriate credit to the original author(s) and the source, provide a link to the Creative Commons license, and indicate if changes were made.
Endoscopic third ventriculostomy ETV and endoscopic to be an effective choice for initial management $[3,4]$.

One of the complications of ETV or ETB is the possible injury to the fornix around the foramen of Monro, resulting in memory impairment. The other complication is bleeding during endoscopic tumor biopsy that could be troublesome and difficult to control [5].

Endoscopic biopsy-related hemorrhage vary in nature from mild tumor hemorrhage to severe intraventricular hemorrhage that necessitate abandonment of ETV if not done earlier and insertion of external ventricular drainage or even shift to microsurgery. Hemorrhage rate vary from approximately 1 to $75 \%$ in different series which can be explained by differences in number of patients 
studied, different tumor pathologies, and surgical technique applied [6-8].

Accuracy of tissue diagnosis through endoscopic biopsy was reported by Baossier et al. to be less than that reported with stereotactic biopsy (81.1\% versus 93.7\%) [9]. The aims of this study were to investigate the safety considerations to minimize tumor biopsy-related bleeding, increase diagnostic accuracy, and establish a successful ETV procedure.

\section{Patient and methods}

This retrospective study includes 25 patients of symptomatic hydrocephalus caused by pineal region tumor operated in Tanta University Hospitals from October 2009 to February 2016. All patients were diagnosed by preoperative MRI images with contrast. The patients' age ranged from 9 months to 65 years with a mean age of 26.5 years.

\section{Preoperative planning}

Preoperative MRI brain with coronal T2- and sagittal T1-weighted images was used to plan the procedure of ETV and tumor biopsy.

The trajectory of ETV was outlined in sagittal images by a line joining tuber cinereum and the anterior edge of the foramen of Monro up to the cortical surface to show up desired burr-hole location.

The trajectory for endoscopic tumor biopsy was outlined by another line from the most proximal part of the tumor to the posterior edge of the foramen of Monro.

If these two presumed burr-holes locations are no more than $1 \mathrm{~cm}$ away, then a single burr-hole for both procedures in a midway location would be appropriate. This single more anteriorly located $(1.5-1.7 \mathrm{~cm})$ precoronal burr-hole was adequate in 17 patients with wide both foramen of Monro and anterior third ventricle, Fig. 1.

With smaller foramen and/or anterior third ventricle, these previously mentioned two lines are away enough, then two separate burr-holes are necessary where an additional burr-hole located more anteriorly $(3-5 \mathrm{~cm}$ from coronal suture) was dedicated for biopsy (eight patients), Fig. 2.

Choice of burr-hole location (right or left) in asymmetric tumor growth is another noteworthy decision. We preferred to approach tumors inclined to the left side through right side burr-hole and vice versa, Fig. 3.

\section{Surgical procedure}

The planned single or double burr-holes were performed. After free-hand insertion of the endoscope, CSF sample is obtained for histopathological examination. We aimed the floor of the third ventricle to perform ETV first. The endoscope is then moved backwards to access the tumor and obtain biopsy in cases of one burr-hole trajectory.

In cases of two burr-holes trajectory, the endoscope is moved completely out for insertion into the biopsy dedicated more anterior burr-hole. Initial coagulation is done over the tumor surface and in cases where tumor is enveloped by ependymal wall to access biopsy underneath the ependyma from within the tumor. A biopsy forceps is used to grasp tumor piece gently moving right and left exerting also a little twist during pull out of the forceps to remove biopsy specimen. At that moment, a French 3 Fogarty balloon catheter is passed through the irrigation channel and put in standby position to do balloon tamponade with inflation of the catheter in biopsy

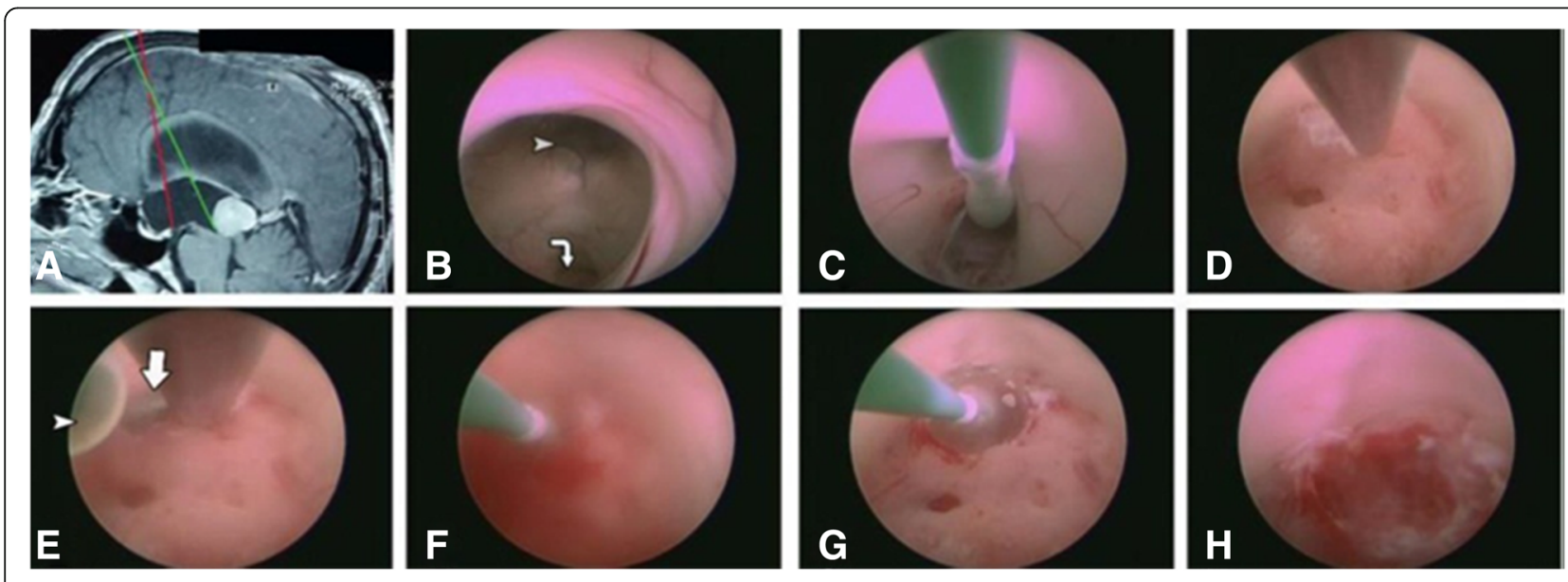

Fig. 1 Shows the single burr-hole method. a Preoperative sagittal T1-weighted image with contrast. The red line represents the pathway for ETV, and the green line represents the pathway for tumor biopsy. $\mathbf{b}$ A view through the foramen of Monro showing both third ventricular floor (arrow head) and pineal region tumor (curved arrow). c Endoscopic third ventriculostomy using Fogarty balloon catheter. $\mathbf{d}$ Initial coagulation on tumor surface. $\mathbf{e}$ Obtaining biopsy from the tumor using biopsy forceps (arrow) where the Fogarty balloon catheter is put in standby position (arrowhead). $\mathbf{f}$ Balloon tamponade to control bleeding from the tumor bed. $\mathbf{g}$ Few minutes after tamponade combined with irrigation showing clear view. $\mathbf{h}$ Final view of clear biopsy bed 

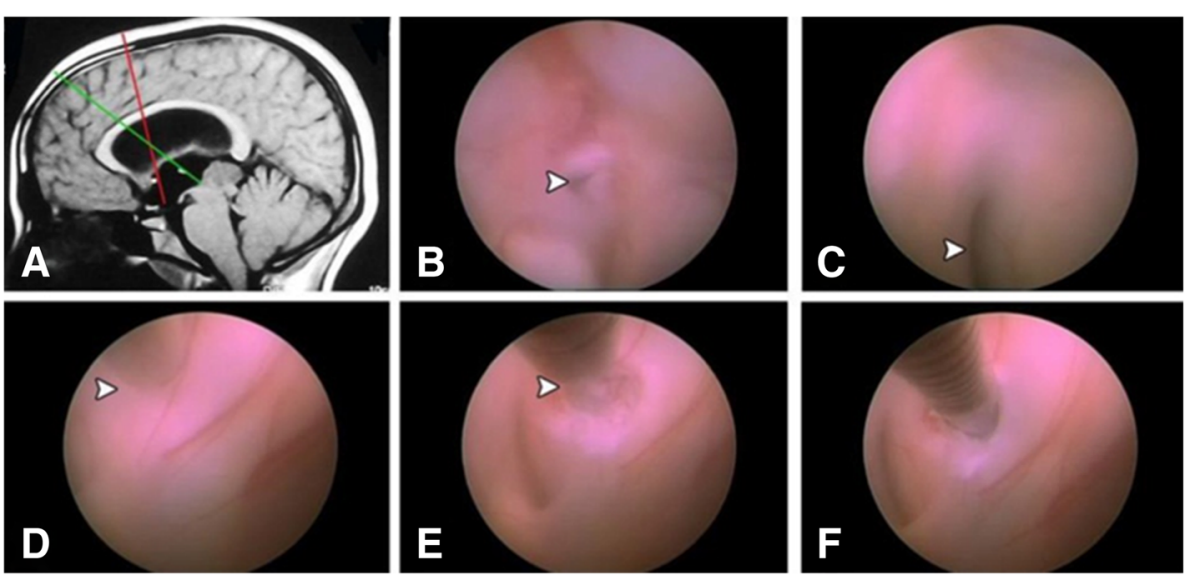

Fig. 2 Shows the two burr-holes method. a Preoperative sagittal T1-weighted image with contrast. The red line represents the pathway for ETV, and the green line represents the pathway for tumor biopsy. The lines are more than $3 \mathrm{~cm}$ away from each other necessitating two separate burr-holes. b Endoscopic third ventriculostomy done first (arrow head). c A close up view through the posterior third ventricle showing pineal region tumor obstructing the aqueduct (arrow head). $\mathbf{d}$ Initial coagulation on ependymal lining. e Biopsy forceps (arrow head) is directed through the cauterized violated ependymal wall. f Obtaining biopsy from within the tumor under ependymal wall using biopsy forceps

bed rapidly controlling bleeding before it could visually obscure the field. The biopsy steps could be repeated to ensure enough tissue submitted for examination. Skin is then closed in layers.

The outcome was verified clinically and radiologically. The mean duration of follow up was 3 years (range $=8-$ 60 months). Follow-up CT images were obtained regularly at 1 and 30 days after surgery. Routine follow-up MRI images were obtained regularly at 3,6 , and 18 months postoperatively. Other follow-up items included the need for any shunt insertion or any neurological complication.

\section{Results}

Twenty five patients of symptomatic hydrocephalus caused by pineal region tumor were included in this study. Sex distribution showed male predominance with 18 cases.
Preoperative clinical manifestations included headache in 22 cases (88\%), papilloedema in 15 cases (60\%), nausea and vomiting in 12 cases (48\%), ataxia in 10 cases (40\%), disturbed consciousness in 4 cases (16\%), and cranial nerve palsy in 4 cases (16\%). Most of these symptoms and signs got significant improvement following the surgery (Table 1).

Headache was the most presenting symptoms in 22 cases (88\%) followed by papilloedema 15 cases $(60 \%)$, nausea and vomiting 12 cases $(48 \%)$, and ataxia in 10 cases (40\%). All the preoperative clinical manifestations were improved postoperatively. Papilloedema, nausea and vomiting, disturbed consciousness, and cranial nerve palsy were resolved completely postoperatively. Headache resolved in 18 cases, improved in 2 cases, and was unchanged in another 2 cases. Ataxia resolved in 90\% of cases and improved in 1 case.

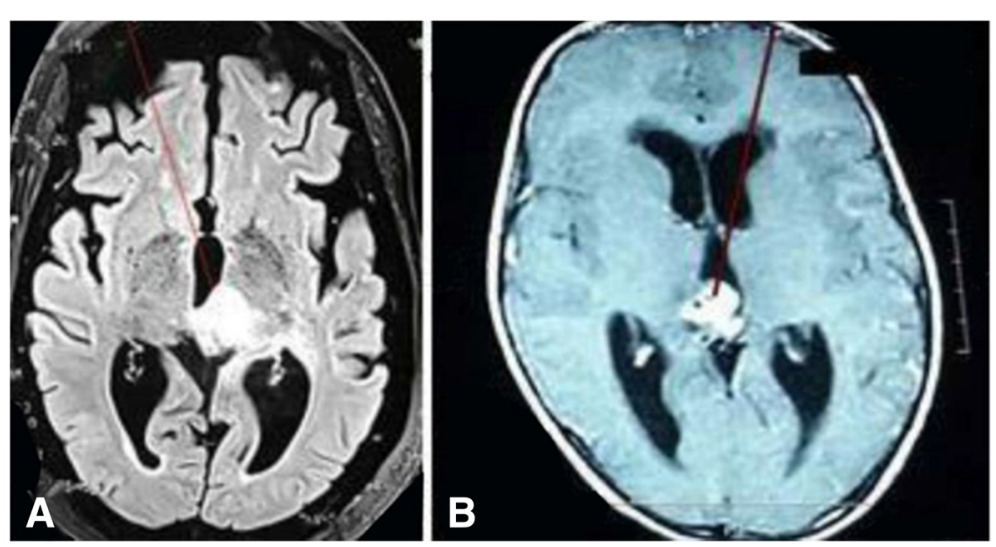

Fig. 3 Shows two examples of left and right inclined tumor and its appropriate approaches. a An example of left side inclined tumor approached through right side trajectory. $\mathbf{b}$ An example of right side inclined tumor approached through left side trajectory 
Table 1 Preoperative clinical presentations and their relative post-operative improvement rates

\begin{tabular}{llllll}
\hline $\begin{array}{l}\text { Clinical } \\
\text { presentations }\end{array}$ & Frequency & $\%$ & Resolved & Improved & Unchanged \\
\hline Headache & 22 & 88 & 18 & 2 & 2 \\
$\begin{array}{l}\text { Papilloedema } \\
\text { Nausea and }\end{array}$ & 15 & 60 & 15 & 0 & 0 \\
$\begin{array}{l}\text { vomiting } \\
\text { Ataxia }\end{array}$ & 10 & 48 & 12 & 0 & 0 \\
$\begin{array}{l}\text { Disturbed } \\
\text { consciousness }\end{array}$ & 4 & 16 & 4 & 0 & 0 \\
Cranial nerve palsy & 4 & 16 & 4 & 0 & 0 \\
\hline
\end{tabular}

Endoscopic third ventriculostomy succeeded in $24 \mathrm{pa}-$ tients resulting in clinical and neuroimaging confirmed improvements in supratentorial ventriculomegaly. One patient failed to improve and eventually required ventriculoperitoneal (VP) shunt. We could obtain endoscopic tumor biopsy from all the patients. No significant intraventricular bleeding reported. The total number of cases required balloon tamponade to control bleeding from tumor bed was 10 cases (40\%).

The CSF analysis show positive cytology only in six cases (24\%). Four cases had germinomas.

A diagnostic tumor biopsy yield was successful in 22 patients. The most common detected histopathological types were germinoma 6 cases (24\%), low grade astrocytoma 6 cases $(24 \%)$, pineoblastoma cases $(24 \%)$, and ependymoma 2 cases (8\%) (Table 2).Germinoma and low-grade astrocytoma were the most common pathological types in 6 cases; $24 \%$ of cases for each of them followed by pineoblastoma 4 cases (16\%) and ependymoma 2 cases (8\%). Central neurocytoma, Dermoid cyst, Metastasis and Pineocytoma had one case (4\%) presentation for each histopathological diagnosis in four cases. Failure to establish a confirmed histopathological examination was reported in three patients with vascular lesions where biopsy specimen was masked by coagulation.

Table 2 Pathological types of the cases

\begin{tabular}{lll}
\hline Pathological types & Number & $\%$ \\
\hline Germinoma & 6 & 24 \\
Low grade astrocytoma & 6 & 24 \\
Pineoblastoma & 4 & 16 \\
Ependymoma & 2 & 8 \\
Central neurocytoma & 1 & 4 \\
Dermoid cyst & 1 & 4 \\
Metastasis & 1 & 4 \\
Pineocytoma & 1 & 4 \\
Undiagnosed & 3 & 12 \\
\hline
\end{tabular}

Definitive treatments for our patients vary from follow up for patient with small low-grade lesions, radiation for all germinoma cases, and surgically unfit patients. Among the remaining patients, four cases accept to perform definitive open microsurgical approach.

\section{Discussion}

The incidence of pineal tumors varies from 1 to $4 \%$ of brain tumors to approximately $11 \%$ in pediatric population. However, only small number of cases has serological diagnostic markers $[9,10]$.

The fore-mentioned fact explains why the initial tumor biopsy is considered as the cornerstone in treatment strategy plan design [2].

Three different techniques are reported in obtaining pineal tumor biopsy. The first is open microsurgical approach which is feasible but associated with morbidity and mortality only in difficult tumor excision like meningioma. The second is the stereotactic biopsy which is considered as a minimally invasive approach with high efficacy and minimal incidence of complication, but it does not offer any help regarding associated obstructive hydrocephalus. And the endoscopic pineal biopsies which provide opportunity to manage hydrocephalus and obtain biopsy under direct visualization at the same time $[9,11,12]$.

Different techniques were described to perform ETV and ETB at the same time. The use of a single compromised (midway) entry site that provide minimal traction on the fornix while performing ETV and minimal traction on the choroid plexus while performing ЕTB $[13,14]$.

The use of flexible endoscope was also applied to easily target ETV and biopsy site but it has low quality image and small working channel if compared to rigid endoscope [15].

Another technique to use two separate navigation designed burr-holes [16]. Morgenstern and Souweidane used the relation between massa intermedia and the tumor as a determinant to perform one or two burr-holes [17].

In this study, we used either single burr-hole or two burr-holes technique based on the preoperative MRI study. The trajectory for endoscopic third ventriculostomy was outlined in sagittal images by drawing a line from the target area in tuber cinereum passing through the anterior edge of the foramen of Monro (fornix) up to the cortical surface to show up desired burr-hole location. The trajectory for endoscopic tumor biopsy was outlined in sagittal images without navigation by drawing a line from the most proximal part of the tumor passing through the posterior edge of the foramen of Monro (fornix) up to the cortical surface to show up desired burr-hole location.

If these two planned burr holes are close, a single more anteriorly located $(1.5-1.7 \mathrm{~cm})$ precoronal burr hole was performed (17 patients). If these two planned burr holes are away enough, then two separate burr holes are necessary where an additional burr-hole 
located close to the hair line $(3-5 \mathrm{~cm}$ from coronal suture) was dedicated for biopsy (eight patients).

We believe that these calculations would be extremely helpful in design of the trajectory to avoid any undue forniceal or third ventricular choroid plexus injury that may potentially complicate the procedure and pose patient at risk. We also believe that in asymmetric tumor growth, it is better to approach tumors inclined to the left side through right side burr-hole and vice versa. This puts the bulging part of the tumor in the viewing angle of the endoscope with wider area to work around and facilitate the process of tumor biopsy.

The CSF analysis of our cases shows positive cytology in only six cases (24\%), four cases of them had germinomas. This highlights the necessary to obtain tumor biopsy in such cases. Another additional benefit of CSF examination in germinoma cases is to determine the need of localized tumor irradiation versus whole craniospinal irradiation.

A potential risk of endoscopic tumor biopsy is bleeding from the tumor bed, especially in these frequently vascular tumors. This bleeding may be initiated suddenly during the pull out of the biopsy sample. Biopsy-related hemorrhage varies from small tumor hemorrhage to severe intraventricular hemorrhage. The incidence of hemorrhage vary in different studies, but it is obviously technique-related, and most experienced surgeon report either no or minimal occurrence of hemorrhage $[6,9]$.

In this study, we used the Fogarty balloon catheter to perform balloon tamponade very early by passing the catheter through the irrigation channel and put it in standby position ready for inflation to control any troublesome bleeding that may initiate during the biopsy. This helps to reduce the operative time and amount of irrigation and/or coagulation required to control bleeding from the tumor bed.

Both endoscopic and stereotactic biopsies for pineal tumors are subject of sample error and needs special care in result interpretation. This may be attributed to heterogenicity of the tumor pathology, amount of tissue supplied for histopathology, and surgical technique applied. No significant difference between both stereotactic and endoscopic approaches was reported. And most studies report success more than $80 \%$ with endoscopic approach $[9,18,19]$.

In this study, pathological diagnosis was obtained in 22 out of 25 cases $(88 \%)$. The reason for difficult histopathological interpretation in the remaining three cases may be attributed to their vascular nature that necessitates excessive coagulation. Although initial coagulation may be helpful to decrease the risk of bleeding, it may risk the histopathological diagnosis by masking the biopsy sample, rendering it difficult to establish a diagnosis.

\section{Conclusion}

Endoscopic tumor biopsy is relatively safe with high diagnostic yield that help in further management protocols plus shunt independent control of associated hydrocephalus.

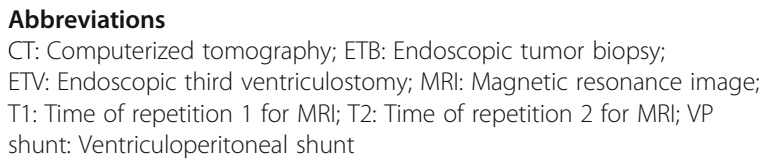

\section{Acknowledgements}

The authors sincerely thank Torky S, ElKheshin S, Saleh A F, and all the department member of Neurosurgery, Tanta University Hospitals for their help in doing this work.

\section{Funding}

There is no funding for this study.

\section{Availability of data and materials}

The datasets used and/or analyzed during the current study are available from the corresponding author on reasonable request.

\section{Authors' contributions}

Both authors equally participate in operating patients, data collection, data analysis and scientific writing. Both authors read and approved the final manuscript.

\section{Ethics approval and consent to participate}

Ethics approval was provided by the ethical committees in faculty of Medicine, Tanta University, Egypt to approve the protocol. Under the number 2981/12/14. Consent for every patient in the study was included.

\section{Consent for publication}

Not applicable

\section{Competing interests}

The authors declare that they have no competing interests.

\section{Publisher's Note}

Springer Nature remains neutral with regard to jurisdictional claims in published maps and institutional affiliations.

Received: 23 October 2017 Accepted: 23 October 2018

Published online: 15 November 2018

\section{References}

1. Robinson S, Cohen AR. The role of neuroendoscopy in the treatment of pineal region tumors. Surg Neurol. 1997:48(4):360-5.

2. Parker JJ, Waziri A. Preoperative evaluation of pineal tumors. Neurosurg Clin N Am. 2011;22(3):353-8.

3. Pople IK, Athanasiou TC, Sandeman DR, Coakham HB. The role of endoscopic biopsy and third ventriculostomy in the management of pineal region tumours. Br J Neurosurg. 2001;15(4):305-11.

4. Morgenstern PF, Osbun N, Schwartz TH, Greenfield JP, Tsiouris AJ, Souweidane MM. Pineal region tumors: an optimal approach for simultaneous endoscopic third ventriculostomy and biopsy. Neurosurg Focus. 2011;30(4):E3.

5. Tirakotai W, Schulte DM, Bauer BL, Bertalanffy $H$, Hellwig D. Neuroendoscopic surgery of intracranial cysts in adults. Childs Nerv Syst. 2004:20(11-12):842-51.

6. Constantini S, Mohanty A, Zymberg S, Cavalheiro S, Mallucci C, Hellwig D, et al. Safety and diagnostic accuracy of neuroendoscopic biopsies: an international multicenter study. J Neurosurg Pediatr. 2013;11(6):704-9.

7. Hayashi N, Murai H, Ishihara S, Kitamura T, Miki T, Miwa T, et al. Nationwide investigation of the current status of therapeutic neuroendoscopy for ventricular and paraventricular tumors in Japan. J Neurosurg. 2011;15(6):1147-57.

8. Wong TT, Chen HH, Liang ML, Yen YS, Chang FC. Neuroendoscopy in the management of pineal tumors. Childs Nerv Syst. 2011;27(6):949-59. 
9. Balossier A, Blond S, Touzet G, Lefranc M, de Saint-Denis T, Maurage CA, et al. Endoscopic versus stereotactic procedure for pineal tumour biopsies: comparative review of the literature and learning from a 25 -year experience. Neuro-Chirurgie. 2015;61(2-3):146-54.

10. Dhall G, Khatua S, Finlay JL. Pineal region tumors in children. Curr Opin Neurol. 2010;23(6):576-82.

11. Sonabend AM, Bowden S, Bruce JN. Microsurgical resection of pineal region tumors. J Neuro-Oncol. 2016;130(2):351-66.

12. Yamini $B$, Refai $D$, Rubin CM, Frim DM. Initial endoscopic management of pineal region tumors and associated hydrocephalus: clinical series and literature review. J Neurosurg. 2004;100(5 Suppl Pediatrics):437-41.

13. O'Brien DF, Hayhurst C, Pizer B, Mallucci CL. Outcomes in patients undergoing single-trajectory endoscopic third ventriculostomy and endoscopic biopsy for midline tumors presenting with obstructive hydrocephalus. J Neurosurg. 2006:105(3 Suppl):219-26.

14. Knaus H, Matthias S, Koch A, Thomale UW. Single burr hole endoscopic biopsy with third ventriculostomy-measurements and computer-assisted planning. Childs Nerv Syst. 2011;27(8):1233-41.

15. Roth J, Constantini S. Combined rigid and flexible endoscopy for tumors in the posterior third ventricle. J Neurosurg. 2015;122(6):1341-6.

16. Kim IY, Jung S, Moon KS, Jung TY, Kang SS. Neuronavigation-guided endoscopic surgery for pineal tumors with hydrocephalus. Minims Invasive Neurosurg. 2004;47(6):365-8.

17. Morgenstern PF, Souweidane MM. Pineal region tumors: simultaneous endoscopic third ventriculostomy and tumor biopsy. World Neurosurg. 2013;79(2 Suppl):S18 e9-3.

18. Azab WA, Nasim K, Chelghoum A, Parwez A, Salaheddin W. Endoscopic biopsy of brain tumors: does the technique matter? Surg Neurol Int. 2014;5:159.

19. Ahmed Al, Zaben MJ, Mathad NV, Sparrow OC. Endoscopic biopsy and third ventriculostomy for the management of pineal region tumors. World Neurosurg. 2015;83(4):543-7.

\section{Submit your manuscript to a SpringerOpen ${ }^{\circ}$ journal and benefit from:}

- Convenient online submission

- Rigorous peer review

- Open access: articles freely available online

High visibility within the field

- Retaining the copyright to your article

Submit your next manuscript at $\boldsymbol{\nabla}$ springeropen.com 